\title{
PERFIL DE USUÁRIOS COM DEMANDA FONOAUDIOLÓGICA EM UM CENTRO DE SAÚDE DE CAMPINAS, SÃO PAULO
}

\author{
Marina Bolsonaro (PG), Núbia Garcia Vianna Ruivo (PQ), Maria Cecília Marconi Pinheiro Lima (PQ), Celso \\ Stephan (PQ).
}

\section{Resumo}

Pesquisa quantitativa com o objetivo de caracterizar o acesso ao serviço de saúde e o perfil dos usuários que possui demanda fonoaudiológica atendidos em um Centro de Saúde de Campinas, SP, realizada a partir do estudo de prontuários. Constatou-se a prevalência de crianças do sexo masculino, encaminhados pelos pediatras com queixa de trocas na fala, com demorado tempo de acesso ao serviço, evidenciando a desproporção entre oferta, capacidade de atendimento e demanda.

Palavras Chave: Fonoaudiologia; Saúde Coletiva; Perfil Populacional.

\section{Introdução}

A identificação do perfil epidemiológico e populacional dos locais onde se nota elevada ocorrência de distúrbios da comunicação é imprescindível para subsidiar o planejamento de ações da fonoaudiologia, sobretudo, na Atenção Básica. Tem potencial para melhorar a qualidade dos atendimentos, identificar as necessidades de determinadas populações e sugerir propostas para resolução de problemas. Dessa forma, o objetivo deste trabalho foi caracterizar o acesso ao serviço de saúde e o perfil dos usuários que possui demanda fonoaudiológica atendidos em um Centro de Saúde da cidade de Campinas, SP.

\section{Resultados e Discussão}

Trata-se de uma pesquisa quantitativa, de caráter descritivo-analítico, realizada a partir do estudo de 168 prontuários de pacientes atendidos pela fonoaudiologia, no período de janeiro de 2009 a setembro de 2014, em um Centro de Saúde de Campinas, SP. O projeto foi aprovado pelo do Comitê de Ética em Pesquisa da Faculdade de Ciências Médicas/UNICAMP sob o número 805.869, aprovado em 23/09/2014.

$\mathrm{Na}$ amostra, houve predominância do sexo masculino (69\%) e prevalência de idade de 0 a 10 anos (63\%). Os encaminhamentos dos usuários para o serviço de fonoaudiologia foram realizados majoritariamente por pediatras $(56 \%)$, com queixa de trocas na fala (36\%). O tempo médio da fila de espera por acolhimento em fonoaudiologia foi de cinco meses. Após avaliação, houve predominância de alterações oromiofuncionais (28\%). É significativa a parcela de usuários que tiveram aleitamento materno (66\%), com duração média de nove meses. Identificou-se, também, presença de hábitos deletérios (mamadeira - 29\% e chupeta - 15\%) entre os pacientes com queixa de alteração oromiofuncional e trocas fonoarticulatórias.
Constatou-se a prevalência de crianças do sexo masculino, encaminhados pelos pediatras com queixa de trocas na fala. Após avaliação fonoaudiológica, os problemas mais recorrentes foram de alterações oromiofuncionais, com significativa presença de hábitos deletérios. O tempo de espera para ter acesso ao serviço de fonoaudiologia é demorado, o que evidencia a desproporção entre oferta, capacidade de atendimento e demanda, trazendo a necessidade de outros profissionais e serviços de fonoaudiologia vinculados à rede de saúde pública, ressaltando a contribuição da integração ensino-serviço.

\section{Agradecimentos}

À família, amigos, orientadores e colaboradores, meus sinceros agradecimentos, visto que de alguma forma doaram um pouco de si para que a conclusão deste trabalho se tornasse possível.

1 ALMEIDA, Sandra Maria Vieira Tristão; REIS, Roberta Alvarenga. Políticas Públicas de Saúde em Fonoaudiologia. In: FERREIRA, Léslie Piccolotto; BEFI-LOPES, Debora M.; LIMONGI, Suelly Cecília Olivan (Org.). Tratado de Fonoaudiologia. São Paulo, SP: Roca, 2010. p. 640-655.

2 BRASIL. MINISTÉRIO DA SAÚDE. Secretaria de Atenção à Saúde, Departamento de Atenção Básica. Cadernos de Atenção Básica: Diretrizes do NASF. Brasília, 2009, 160p.

3 ODK - “Open Data Kit," July 2010. [Online]. Available: http://opendatakit.org/ 